\title{
« Mais où est donc passé mon crédit documentaire? » Acquisition du langage de spécialité
}

Les avatars d'un parcours d'apprentissage dans une classe d'étudiants en commerce international

\section{Marie-Françoise Joncheray}

\section{(2) OpenEdition}

\section{Journals}

Édition électronique

URL : http://journals.openedition.org/asp/3177

DOI : $10.4000 /$ asp.3177

ISSN : 2108-6354

Éditeur

Groupe d'étude et de recherche en anglais de spécialité

Édition imprimée

Date de publication : 1 décembre 1997

Pagination : $317-324$

ISSN : 1246-8185

Référence électronique

Marie-Françoise Joncheray, « « Mais où est donc passé mon crédit documentaire ? » Acquisition du langage de spécialité », ASp [En ligne], 15-18 | 1997, mis en ligne le 13 novembre 2012, consulté le 19 avril 2019. URL : http://journals.openedition.org/asp/3177 ; DOI : 10.4000/asp.3177

Ce document a été généré automatiquement le 19 avril 2019

Tous droits réservés 


\section{« Mais où est donc passé mon crédit documentaire? » Acquisition du langage de spécialité}

Les avatars d'un parcours d'apprentissage dans une classe d'étudiants en commerce international

Marie-Françoise Joncheray

1 Le titre de cette communication peut à première vue paraitre quelque peu provocateur ; en réalité il reflète assez bien le problème que je voudrais traiter ici, celui du signifiant en langue de spécialité qui, si l'on veut pouvoir mettre en place avec succès le lexique et les concepts correspondants en langue étrangère, doit renvoyer à un signifié (ou concept) clairement identifié et maîtrisé en langue maternelle.

2 Je voudrais montrer ici comment, parce que les étudiants n'avaient aucune idée de la réalité que recouvrait l'expression "crédit documentaire», l'apprentissage correspondant en langue étrangère était voué à l'échec.

3 Mais le crédit documentaire, me direz-vous, qu'est-ce que c'est? Peut-être pourrait-on commencer par l'expliquer. Il s'agit d'un moyen de paiement utilisé dans les transactions commerciales internationales afin de se garantir contre le risque de non-paiement des biens vendus. L'importateur met en place un crédit dans la banque de l'exportateur. Le crédit est mis à disposition de l'exportateur contre remise en banque des documents attestant que la marchandise a bien été expédiée, d'où l'expression «Crédit documentaire ». Comme on le voit, il s'agit d'une opération bancaire très technique, tant au niveau des concepts que du vocabulaire employé.

Or, c'est maintenant un lieu commun d'avancer que pour les spécialistes d'autres disciplines, ce n'est pas la langue de spécialité qui pose problème, mais la langue courante, et la relation entre le spécialiste et le linguiste s'exprime bien souvent en ces termes: que le linguiste s'occupe donc de la langue et le spécialiste se chargera de la spécialité. En pratique, les choses ne sont pas si simples. Nous pensons qu'il existe une interaction. Le tout est de savoir quelle interaction. Quelle est en fait la place de l'enseignant de langue en anglais de spécialité? 
5 Nous nous proposons de présenter dans un premier temps les fondements théoriques de notre hypothèse puis, dans un second temps, l'observation qui a suscité cette réflexion, enfin les conclusions auxquelles nous sommes parvenue.

\section{Fondements théoriques}

6 Il existe selon Widdowson (1983) un continuum entre la langue courante et la langue de spécialité: si la langue de spécialité présente des différences telles qu'elle apparait comme une véritable "langue étrangère ", incompréhensible pour le non-initié, il ne s'agit que de différences de contenu, et non de différences de structure.

Comme l'explique Hüllen (1981), ce qui pose problème en langue de spécialité, ce n'est pas l'existence de termes spécifiques, mais les connaissances nécessaires pour comprendre le sens de ces termes. ${ }^{1}$

Il existe une autre différence importante entre la LS et la LC: en LS, le vocabulaire (signifiant) renvoie à un système de concepts (signifié) bien défini et délimité avec précision, ce qui n'est pas les cas en langue courante où le champ sémantique des mots autorise une variété d'interprétations. De ce fait, le lien signifiant-signifié devrait être assez facile à établir, et la compréhension des messages relativement simple.

Le problème est que le mot ne renvoie pas au seul concept, mais aussi à l'ensemble des connaissances qui y sont rattachées et que le sujet a stockées en mémoire. On ne peut réduire la compréhension d'un énoncé au simple repérage des informations qu'il contient, il est également nécessaire d'identifier le domaine concerné par ces informations, et les intégrer dans un système de référence cohérent.

10 Ce qui suppose que l'apprenant confronté à une information en construise une représentation intégrée; il faut, pour qu'il y ait compréhension, pouvoir identifier ce dont on parle. La compréhension d'un message s'accompagne d'inférences déterminées par le contexte, c'est-à-dire par des connaissances extra-linguistiques.

11 Là encore, en langue de spécialité, comme le langage est extrêmement codé et conçu pour être essentiellement un instrument de représentation, le lien entre le sens et les formes linguistiques du discours devrait être relativement simple à établir, contrairement à ce qui se passe en langue courante, où les ambiguïtés créées par l'ampleur du champ sémantique des mots donnent une importance essentielle au contexte pour l'interprétation du message.

12 Nous sommes donc une fois de plus confrontés au problème du sens du langage et de son importance dans l'apprentissage. Widdowson (1974) a montré que les difficultés que les étudiants rencontrent dans leurs productions ne proviennent pas d'une connaissance insuffisante de la langue anglaise, mais de la manière dont le langage peut-être utilisé pour produire du sens au cours des actes de communication. En somme, le problème est de parvenir à une véritable procéduralisation de la connaissance.

Tout part, selon nous, de la manière dont l'input va être intégré par les étudiants, c'est-àdire compris et mémorisé. Au coeur du problème se trouve le contrat tacite qui lie celui qui dispense l'information, locuteur ou enseignant selon le contexte, et celui qui la reçoit, auditeur ou étudiant, et que Haviland et Clark (1974) appellent « given-new contract ».

Lorsque l'information nouvelle qui est la raison d'être du discours est produite, il faut qu'elle soit sous-tendue en quelque sorte par une information ancienne qui va servir de 
référence et permettre au récipiendaire d'identifier le lieu où l'information nouvelle doit s'inscrire dans la présentation déjà construite. Autrement dit, il faut que l'enseignant construise ses énoncés de façon à ce que l'information ancienne puisse effectivement être identifiée comme telle par les étudiants et retrouvée en mémoire. Il faut de plus, pour qu'il y ait apprentissage, que l'influence à construire par l'apprenant pour intégrer la nouvelle information soit la plus simple possible.

La méconnaissance de ces principes a conduit à de graves erreurs pédagogiques même en langue maternelle, lorsque des enseignants tentent de présenter à leurs élèves de nouveaux concepts avec le vocabulaire spécialisé de la discipline. On imagine alors les difficultés lorsque la même situation se présente en langue étrangère et que s'ajoute la nécessité de faire face à un système linguistique plus ou moins bien maîtrisé.

\section{Observation}

Le hasard sert souvent les desseins du chercheur. Les aléas du parcours de formation suivi par notre groupe d'étudiants en commerce international nous ont permis de mettre en évidence l'impact que peut avoir l'existence ou non d'un système de référence procéduralisé dans l'acquisition d'éléments de même nature appartenant à un système linguistique différent.

17 Le public observé est un public captif, composé de 32 étudiants de commerce international, dont 19 ont effectué un stage en entreprise de 8 semaines au moins dans un pays anglophone. L'objet de notre étude concerne l'acquisition de compétences en correspondance commerciale.

18 La méthode d'enseignement suivie dans ce domaine est essentiellement fondée sur les théories de l'apprentissage par observation : on pose que les connaissances sont acquises par l'observation d'un modèle de référence servant de stimulus, selon le jeu de quatre processus : L'attention au modèle observé, la mémorisation, la capacité de reproduire et la motivation.

19 L'acquisition des compétences en correspondance commerciale, discipline extrêmement codée, et qui répond à des normes strictes, nous paraissait particulièrement adaptée à la méthodologie suivie. Même les différences d'ordre culturel existant dans ce domaine ne résistent pas à un travail comparatif systématique entre les productions en langue maternelle et en langue cible. L'apprentissage se fait rapidement et de manière efficace, selon une progression en trois phases (Narcy 1990) : prise des données, production expérimentale, puis transfert et généralisation. Le travail de mémorisation se fait à travers la répétition d'activités permettant le réinvestissement des structures observées.

La motivation des étudiants est fortement instrumentale, en raison des forts coefficients à l'examen à court terme, et à plus long terme du fait que la maîtrise d'une langue étrangère est indispensable pour leur avenir professionnel. La plupart en font d'ailleurs rapidement l'expérience puisqu'ils sont amenés à utiliser la langue étrangère par le biais d'actions commerciales et de stages pratiques qui font partie de leur formation.

21 Appliquée à un public de 120 étudiants réparti en 4 promotions de 30 étudiants suivant un cursus sur deux ans, la méthodologie suivie a donné d'excellents résultats. La première année était consacrée aux acquisitions d'ordre général et culturel, et la seconde aux aspects plus techniques tels que les moyens de paiement, la banque et les assurances. 

faite. Pour comprendre le discours, il n'est pas suffisant de comprendre chaque mot séparément, il faut de surcroît en saisir la cohérence et le sens. Que s'est-il passé au moment de la perception de l'information et de son interprétation dans le traitement de l'information recueillie?

31 Le traitement de l'information résulte du jeu combiné des processus de haut et de bas niveau. Il serait essentiel de comprendre alors l'action de ces processus auxquels nous n'avons qu'un accès réduit et indirect à travers les productions des étudiants.

32 I1 semble bien que, dans le cas que nous avons étudié, les processus de haut niveau (top down) soient restés inefficaces puisque le discours proposé ne correspondait à aucun 
schéma connu des étudiants. Tout se passe comme si, en état de surcharge cognitive, les processus de haut niveau "disjonctent». Daniel Gaonac'h parle de "court-circuit» lorsqu'il se réfère aux processus mis en oeuvre lors de la lecture d'un texte en langue étrangère: les insuffisances linguistiques ont pour effet de "court-circuiter » les processus de haut niveau, et même les bons lecteurs en langue maternelle ont alors recours à des stratégies de bas niveau. D'où la nécessité pour le pédagogue de mettre en place des tâches qui vont faciliter l'action des processus de haut niveau. Gaonac'h fait la même remarque en ce qui concerne les connecteurs logiques en langue étrangère : un trop grand nombre de marqueurs constitue une surcharge en compréhension orale.

Dans le cas que nous étudions, la surcharge est double: il s'agissait pour les étudiants d'assimiler des concepts nouveaux, et de le faire avec un vocabulaire nouveau. Devant l'accumulation de données linguistiques, les processus de haut niveau se bloquent. La compréhension ne peut alors avoir lieu, et la production devient ipso facto impossible.

Nous pensons que cette observation est à rapprocher du problème du sens en apprentissage. Pour que l'apprentissage se fasse, il faut que son objet ait un sens pour l'apprenant. Si le sens n'est pas établi, il n'y a pas d'apprentissage. Ceci nous paraît important pour l'acquisition d'une langue étrangère à tous niveaux: l'acquisition linguistique sera difficile en l'absence d'un système de référence construit, et à l'inverse, l'acquisition du système de référence implique que la langue soit acquise, elle doit donc se faire en langue maternelle. Vygotsky (1962) explique que c'est à travers le langage que la conceptualisation se fait. Les concepts ne peuvent être élaborés sans les mots, on ne peut construire un système conceptualisé sans l'outil du langage. Ainsi donc, on ne peut introduire des notions nouvelles avec du vocabulaire nouveau. Or il est nécessaire qu'un niveau de conceptualisation suffisant soit atteint en langue maternelle ou en langue 2, pour permettre l'introduction de nouvelles données. Ce qui nous amène à penser que lorsque l'étudiant apprend une spécialité, il est préférable que la conceptualisation soit effectuée par l'intermédiaire de la langue maternelle pour permettre l'acquisition de ces mêmes notions en langue étrangère.

Ceci nous montre que, pour être efficace, l'enseignement d'une langue de spécialité doit donner lieu à un véritable travail d'équipe entre le spécialiste et le professeur de langue, de façon à construire une progression ordonnée et à favoriser la mise en place des concepts en langue maternelle avant l'intervention du linguiste. En effet, il est essentiel que les étudiants puissent construire leur système de référence à l'aide d'un vocabulaire qu'ils maîtrisent. Ils seront alors à même de relier le signifiant linguistique à un concept identifié en langue maternelle, et l'apprentissage en langue étrangère deviendra possible.

\section{BIBLIOGRAPHIE}

Allen, J.P.B. \& H.G. Widdowson. 1987. « Grammar in Language Teaching ». In Hutchinson, T. \& A. Waters, English for Specific Purposes. Cambridge : Cambridge University Press.

Caron, J. 1989. Précis de psycholinguistique, Paris : PUF, 1989. 
Gaonac'h, D. 1990. «Les stratégies attentionnelles dans l'utilisation d'une langue étrangère ». Le Français dans le monde, EDICEF février-mars.

Hüllen, W. 1981. « The teaching of English for specific purposes : a Linguistic view ». In Freudenstein, R. et alii (dir.), Language Incorporated : Teaching Foreign Languages in Industry. Oxford : Pergamon Press.

Hutchinson, T. \& A. Waters. 1987. English for Specific Purposes. Cambridge : CUP, 1987.

Kess, J. F. 1992. Current Issues in Linguistic Theory. Psycholinguistics. Amsterdam : John Benjamins Publishing Company.

Narcy, J-P. 1990. Apprendre une langue étrangère. Paris : Éditions d'organisation.

Vygotsky, L.S. 1992. « Thought and Language ». In Kess, J. F., Current Issues in Linguistic Theory. Psycholinguistics. Amsterdam : John Benjamins Publishing Company.

Widdowson, H.G. 1983. Learning Purposes and Language Use. Oxford : Oxford University Press.

\section{ANNEXES}

\section{Annexe 1}

\section{Texte à mettre en anglais}

Conditions de paiement pour une première commande de l'étranger = comptant contre documents. Connaissements et certificat d'assurance contre paiement de la facture à la Barclay's Bank - York.

\section{Erreurs concernant la compréhension du fonctionnement du contenu documentaire}

* Cash against documents of consignment and certificate of insurance against payment of the invoice.

* We usually deal with a cash discount against a bill of lading and an insurance certificate on a document against acceptance in payment of the invoice to the Barclay's Bank in York.

* the bill of lading will be sent to you...

* bill of lading and insurance certificate accepted against payment of the invoice...

* The bill of lading and insurance certificate have to be sent against payment of the invoice...

*Même phrase avec have to be accepted

*cash document against bill of lading and insurance certificate given against settlement of the bill...

* We demand to be settled cash, documents against acceptance.

* We would like to inform you that we require you to pay at sight against documents. As soon as we receive your statement, the bill of lading and the insurance certificate will be sent to you;

* We usually deal with a cash discount against documents, documents against acceptance and insurance certificate against payment of the invoice.

\section{Annexe 2}

Erreurs de lexique

Pour bill of lading: *documents of consigument, the sea-bill [sic] 
Pour cash against documents : *a cash discount, documents against acceptance, documents against cash, cash against acceptance

Pour invoice : *bill

Pour certificate of insurance : *insurance policy

Pour settlement : *statement

Pour the documents will be handed over to you : * the bill of lading will be delivered to you, the bill of lading will be transfered to the bank, the remittance of the bill of lading

Pour we usually deal on a cash against documents basis : * we are used to dealing with a cash against document, we deal with cash against documents, we accept payment by using cash against documents

\section{NOTES}

1. "It is not the usage of technical terms per se which distinguishes language for special purposes from general language, but the factual knowledge necessary for understanding these words ».

\section{RÉSUMÉS}

L'observation des parcours d'apprentissage dans une classe de BTS Commerce international a permis de mettre en évidence la nécessaire présence d'un système de référence construit en L1, pour permettre l'acquisition de ces mêmes notions en langue étrangère. En l'absence d'un tel système, l'apprentissage ne se fait pas.

If students are to acquire the specialised language they need in a foreign language for their profession, they must first have acquired the necessary specialised knowledge in L1. Failing that, acquiring the corresponding foreign language will be made very difficult.

\section{INDEX}

Mots-clés : apprentissage, cognition, langue étrangère, pédagogie, système de référence Keywords : foreign language, learning, reference system, teaching

\section{AUTEUR}

\section{MARIE-FRANÇOISE JONCHERAY}

Marie-Françoise Joncheray est professeur agrégé d'anglais au lycée Carcouet de Nantes. Elle intervient également en formation des maîtres à l'IUFM de Nantes. Elle prépare une thèse en didactique des langues à l'Université de Technologie de Compiègne sous la direction du professeur Jean-Paul Narcy.mf.narcycombes@wanadoo.fr 